\title{
Integrating computer-aided modeling and micro-simulation in multi-criteria evaluation of service infrastructure assignment approaches
}

\author{
Alfonso Duran, Isabel Garcia, Miguel Gutierrez \\ Univ. Carlos III de Madrid (Spain) \\ duran@ing.uc3m.es, igarcia@ing.uc3m.es, mgfernan@ing.uc3m.es
}

Received: September 2012

Accepted: March 2013

\section{Abstrad:}

Purpose This paper proposes an integrated computer-supported multi-staged approach to the flexible design and multicriteria evaluation of service infrastructure assignment processes/ algorithms.

Design/methoddogy/approadr It involves particularizing a metamodel encompassing the main generic components and relationships into process models and process instances, by incorporating structural data from the real-life system. Existing data on the target user population is fed into a micro-modeling system to generate a matching population of individual "virtual" users, each with its own set of trait values. The micro-simulation of their interaction with the assignment process of both the incumbent and the competitors generates a rich multidimensional output, encompassing both "revealed" and non-observable data. This enables a comprehensive multi-criteria evaluation of the foreseeable performance of the designed process/ algorithm, and therefore its iterative improvement.

Findings: The research project developed a set of methodologies and associated supporting tools encompassing the modeling, micro-simulation and performance assessment of service infrastructure assignment processes. 
Originality/value The proposed approach facilitates, in a multicriteria environment, the flexible modeling/design of situation-specific assignment processes/algorithms and their performance assessment when facing their case-specific user population.

Keywards: infrastructure assignment, metamodeling, microsimulation, multicriteria, simulation platforms

\section{Introduction and Objectives}

The assignment of usage slots of fixed capacity, non-storable service infrastructures (such as nights at a hotel rooms or utilization of a surgery operating table or medical emergency resource) has both significant economic relevance and methodological complexity. Faced with heterogeneous potential users, the realization of the infrastructure's potential value depends on its actual utilization and on the appropriateness of the match with the actual users it is assigned to. Unused slots create no value, and the value of assigned slots might be very different depending on the specific assignment, as illustrated by the widely different prices often paid for contiguous seats in the same plane.

These allocation decisions, characterized by negligible variable (volume-related) costs in comparison with fixed costs, "perishable" resources and heterogeneous potential customers can gain insights from the application of "Revenue Management" (RM) approaches (Chiang, Chen \& Xu, 2007; Abdelghany \& Abdelghany, 2008). Given essentially fixed cost and adopting profit maximization as the sole objective, RM focuses on extracting the maximum revenue from a stream of potential customers, with different price sensitivity, within existing resource constraints. Thus, RM applies price discrimination, selling essentially the same service to customers in different "rate classes" at different prices.

RM approaches, however, have two major limitations. Ad-hoc development or adaptation of assignment algorithms is required to tailor them to each organization's specific structural situation (assignment process design,...). This might be cumbersome and results in untried procedures, particularly given the particularities of each organization's user population. Additionally, sole focus on direct, short-term revenue/ profit maximization is often excessively narrow.

This paper presents an integrated approach to tackling these issues. Thus, the major objectives of the approach discussed here are:

- Support the flexible modeling/design of situation-specific assignment processes/algorithms by incorporating specific structural data to a generalized model ("metamodel") of infrastructure assignment. 
- Facilitate credible assessment of the performance of the resulting designs taking into account the peculiarities of that situation's user population.

- Enable appropriate multicriteria analysis.

This approach and the enabling tools have been developed within a 4-year publicly funded research project with the support of four collaborating organizations.

\section{Structure of the Integrated Approach}

Figure 1 depicts the general structure of the integrated approach proposed in this paper. This section contains a general description of the approach, its components, relations and support tools, which are then discussed in subsequent sections.

For infrastructure assignment decisions, in coherence with the first objective, a generalized metamodel has been developed, along with a methodology supported by a proof-of-concept computer tool to enable the flexible modeling/design of case-specific assignment processes/algorithms by incorporating the structural data from the real life system's infrastructure assignment business process. This component is further discussed in the following section.

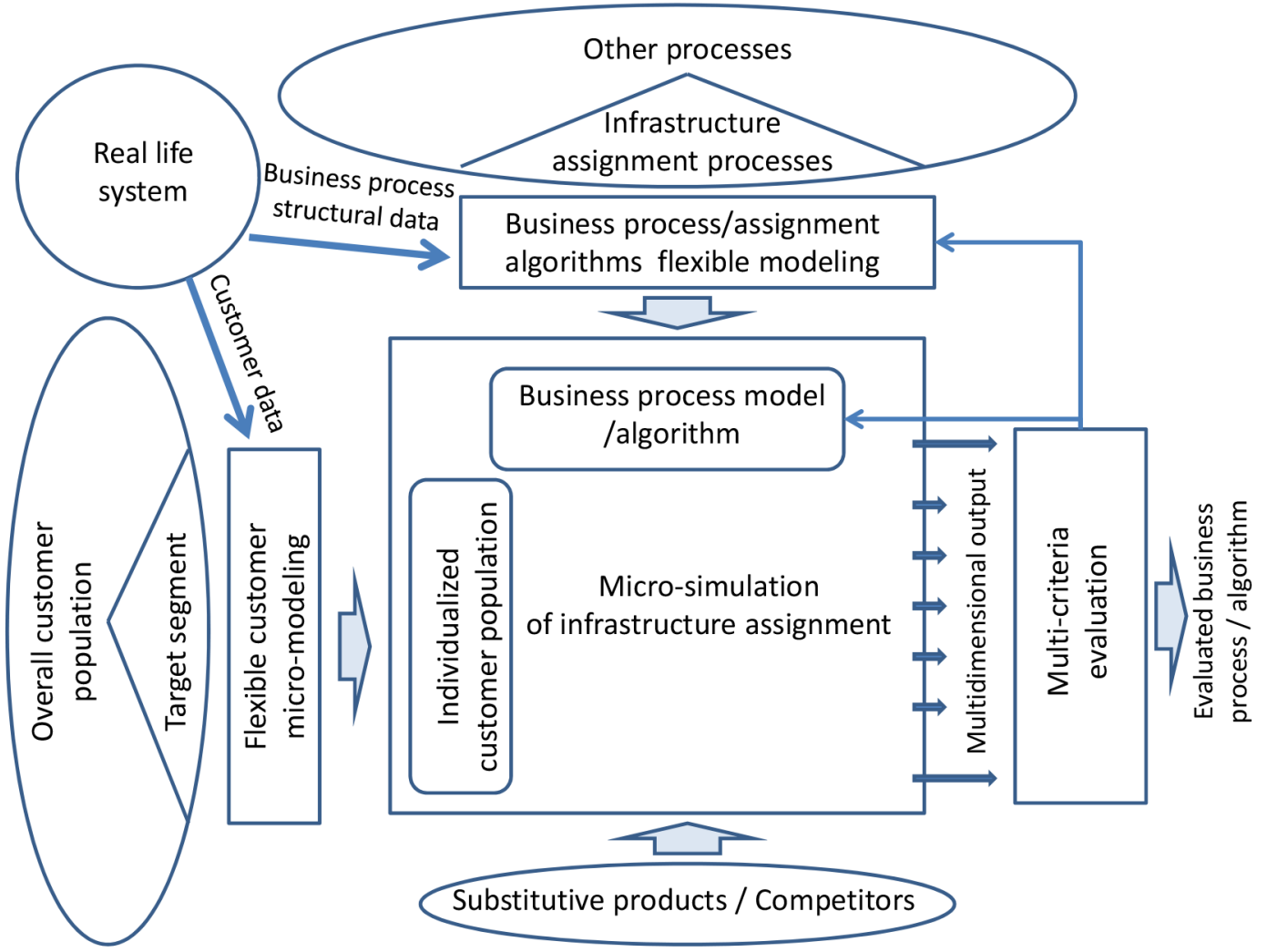

Figure 1. Structure of the proposed integrated approach

In order to take into account the peculiarities of each situation's user population, all existing customer data for the real system's target segment is incorporated into a flexible customer 
micro-modeling system, leading to the generation of a population composed of individual "virtual" customers, each with its own set of trait values, that closely resembles the real system's user population.

These individualized users are loaded into an ad-hoc micro-simulation platform, where they face both the assignment business process/algorithm designed for the incumbent organization and a simplified representation of the substitutive products/competitor's offerings, as discussed in section 4.

The micro-simulation of the interaction between the user population, the incumbent's assignment process and that of the competitors generates a rich multidimensional output, encompassing both "revealed" data, that would be observable in real life, and non-observable data. This enables a comprehensive multi-criteria evaluation of the foreseeable performance of the designed business process/ algorithm, that can be used for its iterative improvement, as discussed in section 5 .

\section{Business process/assignment algorithms flexible modeling}

As outlined in the previous section, following the characterization of the infrastructure assignment business processes that are the focus of this research, these target processes have been analyzed to synthesize the generic model that encompasses them. This generic model of business process models, or business process metamodel, includes the main generic components of the infrastructure assignment models as well as their relationships (OMG, 2006).

Figure 2 depicts the UML class diagram of the proposed metamodel (Gutierrez \& Duran, 2011). An infrastructure assignment process model defines how a particular type of infrastructure is assigned to one of its potential uses. Customers grouped by customer segments (Customer Segment Type) demand the use of specific infrastructures (Infrastructure Type) through defined access channels (Channel Type). In the most general case, all Customer Segment Types would be able to access all Infrastructure Types through all Channel Types; the existence of restrictions in the access to some Customer Segment Types, or access to certain infrastructures through some Channel Types, will lead to a complete set of problems to be addressed.

Each combination of possible types of infrastructure access (Infrastructure Access Type) might be assigned different Value Types. In commercial infrastructure allocation problems, these Value Types will be the tariff types, e.g. reduced tariff, normal tariff, premium tariff; offer price, list price; high season, low season... The assignment of a Value Type to an Infrastructure Access Type defines a generic Allocation Type. Each Allocation Type will apply during a time interval, which will be defined by a Generic Start Time and a Generic End Time. These times are handled in the generic model layer in abstract form, without taking a specific 
value or time. In some complex models logical relationships among them (Constraints) will have to be defined. According to this approach, a Business Process Model is defined as a set of Allocation Types.

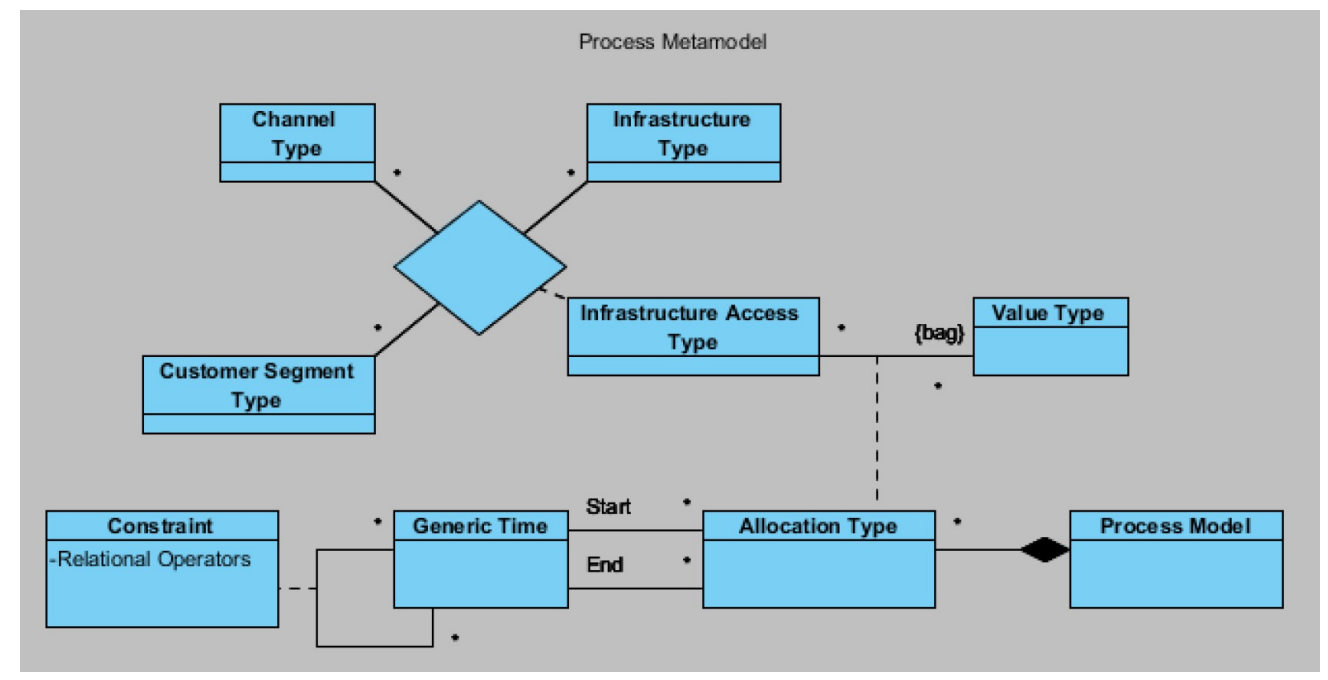

Figure 2. UML class diagram of the Process Metamodel

Each Process Model would generally yield to a number of Process Instances. Conceptually, process instances can be identified as infrastructure assignment optimization problems.

A computer tool which has been built upon the described metamodel assists in structuring real life system data in order to define infrastructure assignment process models and corresponding process instances through a guided sequence of windows-based forms. It contains a representative collection of internally defined possible parameter and variable types. There is also a procedure that automatically identifies forecasting requirements for those attributes stated as statistical parameters, such as the mean of the distribution of the customer segment's arrival rate.

In a second set of windows-based forms, the computer tool helps the definition of the process instance to be considered in a specific case, i.e., the assignment business process to be designed for the incumbent organization. Entity attributes are defined for each instance, specifying if they should be stated as variables. These attributes are loaded in the corresponding database in an automatic pre-compiled procedure. Lastly, the tool assists in the definition of multi-objective functions, through mathematical combinations of a subset of parameters and variables.

\section{Micro-modeling of the target user segment and micro-simulation platform}

In the multi-staged integrated approach proposed in this paper, the initial assignment process/algorithm/forecasting requirements designed with the computer-aided methodology described in the previous section analyze the user population only at an aggregated level, as being composed of sub-segments characterized by attributes such as arrival distribution parameters. To accomplish the second objective (credible performance assessment taking into 
account the peculiarities of each situation's user population) and to enable the fulfillment of the third (multicriteria evaluation), a computer-supported methodology has been developed for the micro-modeling of the target user segment and the corresponding micro-simulation of the assignment process (Klevmarken, 2002; Abdelghany \& Abdelghany, 2008).

Thus, users are now modeled as individual "virtual" software agents, each with its own set of trait values. The methodology calls for the introduction, through series of menus and input tables, of all available data on the characteristics of the target users in the real-life system for which the assessment process is being designed. This leads to the generation of an individualized representation of the target user segment in a way that fully exploits all existing user information to ensure that it resembles the real system's user population as closely as possible, in two key dimensions: the internal correlation of the traits (i.e., which traits tend to happen together) and the average value distribution for each individual trait.

These individualized users are loaded into an ad-hoc micro-simulation platform, where they face both the assignment business process/algorithm designed for the incumbent organization and a simplified representation of the substitutive products/competitor's offerings. The match between the traits of each individual user and the characteristics of each service infrastructure alternative will influence the attractiveness for that particular user of each alternative. As an illustration of the platform's design, Fig 3 shows a subset of the UML class diagram highlighting the generation of this flexible "attractiveness" or "utility function", named "Willingness To Pay" in the platform but fully generic in nature.

The system's behavior is then simulated as the aggregated effect of these individual decisions over time. As discussed in the next section, this approach, besides its potential for a more accurate representation of the system's aggregated evolution, allows data access, for analysis and evaluation purposes, not only at a disaggregated level but also for data dimensions that would be non-observable in other approaches.

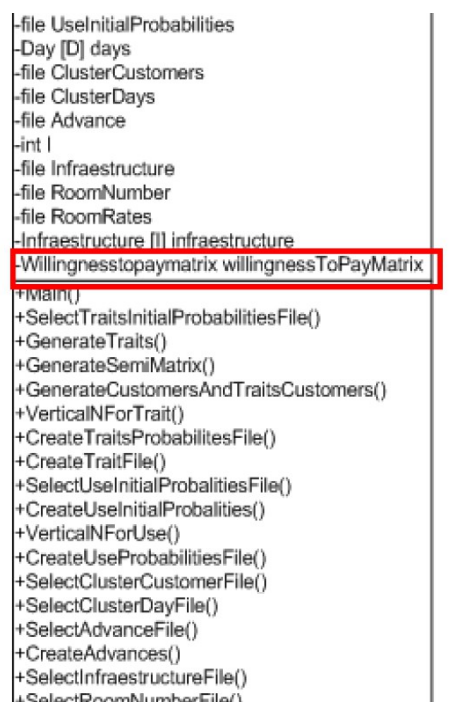
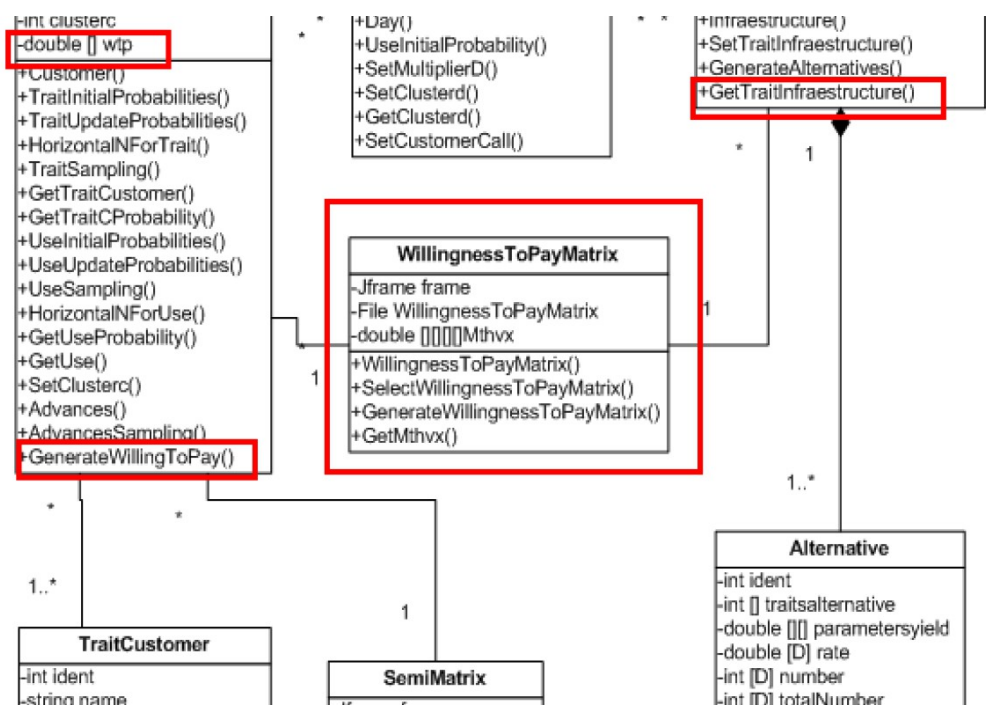

Figure 3. Subset of UML class diagram highlighting the generation of the "Willingness to pay" 


\section{Multidimensional output and multi-criteria evaluation}

Micro-simulation provides a wide and rich set of output variables that may be used to compare the performance of alternative business process designs, as well as for their further refinement. Modeling customer behavior traits results in two main types of output variables:

- Revealed data. This is the type of data that could actually be registered in a real-life situation, since it is observable by the hotel, like room occupation levels per room type and day, or daily revenue per room type. In real-life cases, even if this data is recordable, it may be only partially recorded, as the required information system and procedures may or may not be implemented.

- Non-revealed data. These are typically customer traits, highly relevant for business process performance, but which may not be easily observed, e.g. the maximum price that a particular customer is ready to pay for a good/service (Willingness To Pay). If the necessary information is available, the simulation platform assigns values for these traits to each individual user, thus providing a useful output for performance assessment. For example, hotel customers may be clustered in price categories according their willingness to pay, and by comparing these figures with the actual assignment performed, the effectiveness of the assignment process may be assessed.

These two sets of data are used to create the dimensions of the multi-criteria evaluation for the alternative designs considered. In some sectors, such as health care, multi-criteria evaluation has an immediate fit. Furthermore, even in sectors where profit is the main performance criterion, a multi-criteria approach may also be useful. Mid and long-term revenues are often dependent on aspects such as customer loyalty or the company image, as projected through the experience of customers, via e.g. comments in web forums. These elements, that directly or indirectly impact revenue, can be organized along the following evaluation dimensions:

- Current revenue obtained from the assigned infrastructure.

- Future revenue streams, which may be subdivided into three components:

- Customer loyalty/repetition. These indicators are related to the repetition by the same customer, and are influenced by such aspects as the customer's perception of the gap between service received and service expected.

- Influence on potential customers. Indicators related to the influence that the customer's perception may have on other potential customers, for instance through comments introduced into a web site.

- Related goods or services (cross-selling). Indicators related to the likelihood that various types of customers generate additional revenue through the consumption of 
products or services related to the assigned infrastructure, e.g. expenditures on the hotel's ancillary services.

In sectors where the objective function is intrinsically multi-criteria, e.g. health, in spite of the importance of economic criteria the evaluation context is always more complex as social aspects play a critical role, and a multi-criteria approach for evaluation is likely to be the most appropriate solution (Bana-Costa, Fernandes \& Correia, 2006).

This diversity among sectors in the assessment criteria prevents the prescription of a single multicriteria method. Instead, a flexible approach is needed to choose the most appropriate method for each case. For instance, in sectors where the main evaluation criteria are economic, a method like AHP may be suitable. This method is intuitive for the user and provides a very good capability for criteria structuring in successive hierarchical levels. In the case of sectors more intrinsically multicriteria, it may be more appropriate to use a more sophisticated non-compensatory method, for instance, ELECTRE or PROMETHEE (Pomerol \& Barba-Romero, 2000).

\section{Conclusions}

The integrated computer-supported multi-staged approach to the flexible design and multicriteria evaluation of service infrastructure assignment processes/ algorithms proposed in this paper tackles the main limitations limiting the applicability of Revenue Management "conventional" approaches, namely their requirement of cumbersome, untried ad-hoc adaptations to cater to each organization's specific structural situation, and its narrow focus on direct, short-term profit maximization.

By way of the successive particularization of a generalized metamodel into process models and process instances by incorporating structural data from the real-life system, it supports the flexible modeling/design of situation-specific assignment processes/algorithms. By feeding existing data on the target user population into a micro-modeling system to generate a population of individual "virtual" users, each with its own set of trait values, that closely resembles the real system's users, it takes into account its peculiarities. Through the rich multi-dimensional output generated by the micro-simulation of the interaction of this individualized population with the assignment process of both the incumbent and its competitors, it enables a comprehensive multi-criteria evaluation of the foreseeable performance of the designed process/ algorithm, and thus its iterative improvement.

\section{Acknowledgements}

This work stems from the participation of the authors in a research project funded by the Spanish National Research Plan, reference DPI2008-04872, title "Optimizacion de la asignación de infraestructuras de servicios mediante simulación - sectores hotelero y sanitario" 


\section{References}

Abdelghany, A., Abdelghany, K. (2008). A micro-simulation approach for Airline Competition Analysis and Demand Modelling. International Journal of Revenue Management, 2(3), 287-306. http://dx.doi.org/10.1504/IJRM.2008.020626

Bana-Costa, C.A., Fernandes, T.G., Correia, P.V.D. (2006). Prioritisation of public investments in social infrastructures using multicriteria value analysis and decision conferencing: a case study. International Transactions in Operational Research, 13(4), 279-297. http://dx.doi.org/10.1111/j.1475-3995.2006.00549.x

Chiang, W.C., Chen, J.C.H., Xu, X. (2007). An overview of research on revenue management: Current issues and future research. International Journal of Revenue Management, 1(1), 97-128. http://dx.doi.org/10.1504/IJRM.2007.011196

Gutierrez, M., Duran, A. (2011). Generic Model Base Design for Decision Support Systems in Revenue Management: Applications to Hotel and Health Care Sectors. In C. Jao (ed.) Efficient Decision Support Systems: Practices and Challenges-From Current to Future, 183-194.

Klevmarken, N.A. (2002). Statistical inference in micro-simulation models: incorporating external information. Mathematics and computers in simulation, 59(1.3), 255-265. http://dx.doi.org/10.1016/S0378-4754(01)00413-X

OMG - Object Management Group (2006). Meta-Object Facility (MOF) V 2.0 core specification. http://www.omg.org/spec/MOF/2.0/PDF. Accessed 23 Feb 2012.

Pomerol, J.C., Barba-Romero, S. (2000). Multicriterion Decision in Management: Principles and Practice. Kluwer Academic Publishers, Norwell.

Journal of Industrial Engineering and Management, 2013 (www.jiem.org)

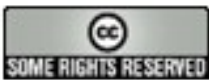

Article's contents are provided on a Attribution-Non Commercial 3.0 Creative commons license. Readers are allowed to copy, distribute and communicate article's contents, provided the author's and Journal of Industrial Engineering and Management's names are included. It must not be used for commercial purposes. To see the complete license contents, please visit http://creativecommons.org/licenses/by-nc/3.0/. 